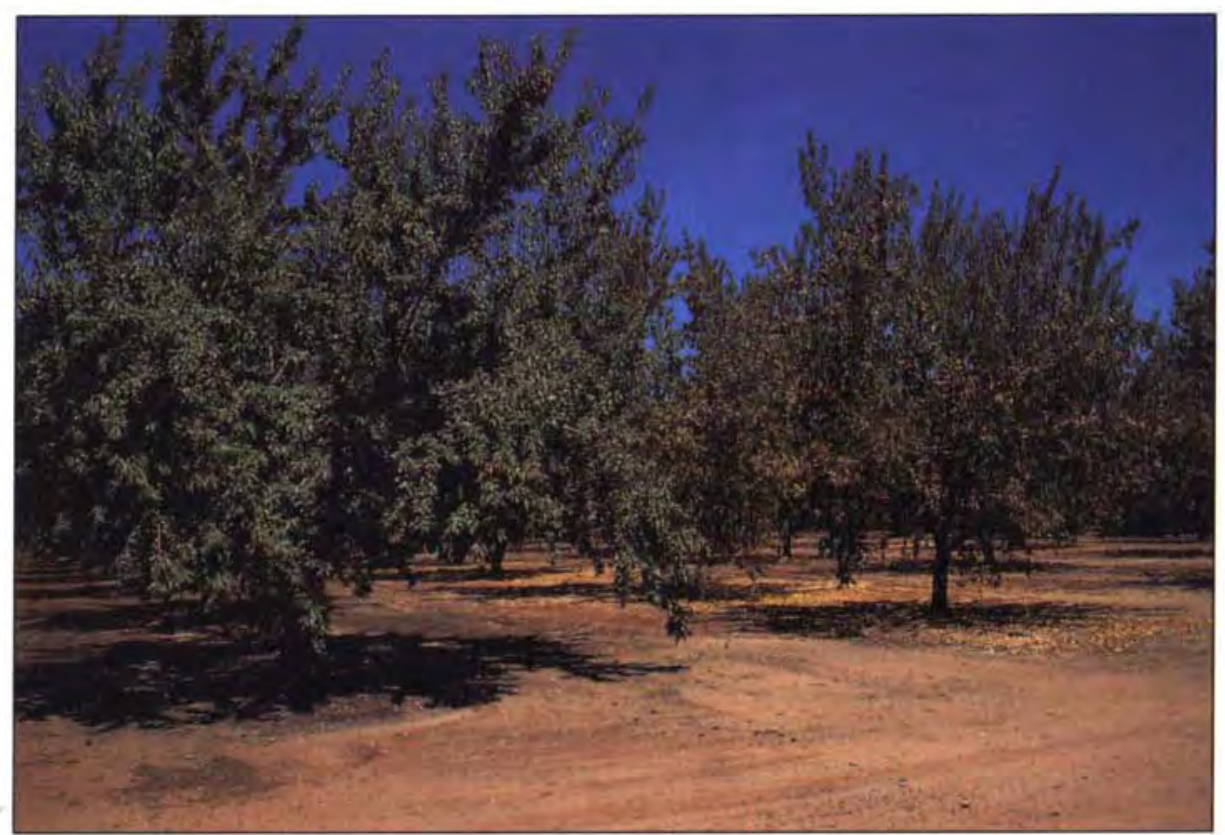

Above, the incidence of almond hull rot was affected by the timing of irrigation cut-off and hull split size, but not by inoculum concentration. The incidence of hull rot was significantly higher in trees irrigated within 2 weeks of harvest (the greener trees shown above). Right, almond yields are reduced by hull rot, which destroys the fruiting wood.

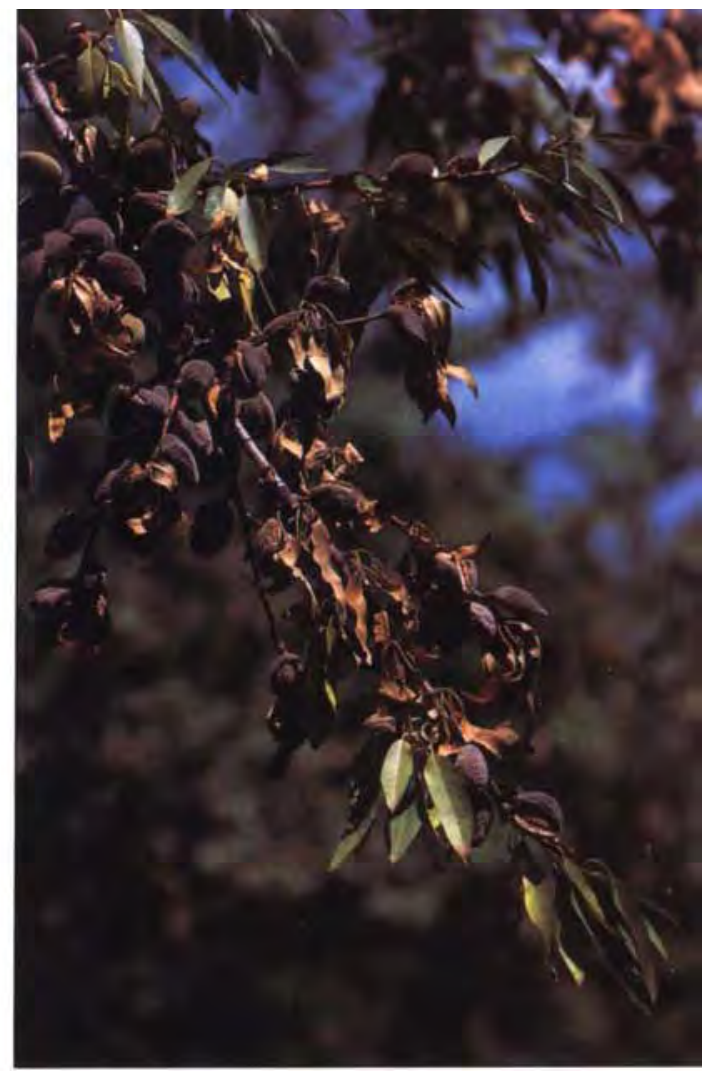

\title{
Cutting off irrigation early may reduce almond hull rot
}

\author{
Beth L. Teviotdale a Themis Michailides a David A. Goldhamer \\ Mario Viveros $\square$ Lisa Schmidt $\square$ Valeshia Hines
}

\section{Cutting off irrigation two weeks before harvest substantially re- duced the damage caused by hull rot in a Kern County almond or- chard trial. The extent of leaf death was affected by the stage of hull split at which the fruit were inoculated, but not by the concen- tration of inoculum.}

Hull rot is a sporadic but sometimes serious disease of almond, Prunus dulcis (Mill) D. Webb, in California. It is caused by Rhizopus stolonifer (Ehrenb:Fr.) Vuill. or Monilinia fructicola (G. Wint.) Honey. Three other fungi, $R$. circinans Tiegh, $R$. arrhizus $\mathrm{A}$. Fischer and M. laxa (Kaderhold \& Ruhland) Honey, can cause hull rot symptoms, but rarely are found associated with the disease in the field. Although all almond cultivars are susceptible, the widely planted 'Nonpareil' cultivar is the most susceptible. Vigorous, heavily cropped orchards usually sustain the greatest damage, and no control measures have been defined.

The first symptom of hull rot is a grayish lesion, which soon turns tan to brown on the hull of the maturing almond fruit. Then the nearby leaves and part or all of the spur or shoot upon which the infected fruit is borne begins to die, probably due to a toxin produced by the pathogens and transported from the infected hull to the spurs and leaves. The vascular tissues leading to the infected fruit die and turn black. The dead leaves remain attached to the twigs, imparting a scorched appearance to badly damaged trees. Sporulation by Rhizopus is easily visible between the hull and shell, and $M$. fructicola often can be found sporulating on inner and outer hull surfaces. Although the edible nut is not harmed, yields are reduced because hull rot destroys the fruiting wood.

Since pathogens cannot invade the outer hull surface of the almond fruit, hull rot infections occur during hull split, when the hull opens along the suture. Infection starts as the fungal spores, probably carried by insects and wind currents, are deposited on the inner hull surface. The period of potential infection corresponds to the hull split process: Hull split begins in early July in 'Nonpareil' trees planted in the southern San Joaquin Valley; 
tory. Hulls were considered split if any part of the suture had separated 0.04 inch or more. Hulls were separated from the nuts, weighed, then airdried in a forced-air oven at $150^{\circ} \mathrm{F}$ for 72 hours to determine dry weight. Percentage hull moisture content was calculated from these figures.

There were three replications of each irrigation cut-off treatment, arranged in a randomized complete block design, and each plot consisted of eight rows 12 trees long. Data were collected from the center four pairs of 'Nonpareil' trees within each plot.

Hull rot was studied in two ways, by measuring natural infection and conducting inoculation experiments.

Natural infection. We measured the incidence of natural infection at harvest in 1990 and 1991. Trees were shaken on August 16 in both years. Immediately afterwards, strikes were counted (strike designated a short spur with one or two clusters of dead leaves or a single cluster of dead leaves on a shoot), and the inches of dead wood present were estimated in all eight data trees in each replication. Random samples of fruit were collected from the ground beneath the trees, placed in paper bags, and transported to the laboratory, where 100 fruit were drawn from each sample and examined for presence of hull lesions and growth of hull rot fungi.

Hull split size and inoculum concentration. We studied the effects of hull split size and inoculum concentration on incidence of hull rot in 1991. Inoculum was prepared from cultures of one isolate of $R$. stolonifer obtained from almond. Approximately 0.1 to 0.2 $\mathrm{ml}$ of inoculum was introduced into the fruit through open sutures with an artist's airbrush. No water-soaking or other damage to the hull tissues was observed. We inoculated 25 fruit per replication at approximately weekly intervals on each of four dates, beginning at early hull split and ending within 2 weeks of harvest. The inoculated fruit were numbered and their locations identified with flagging tape. Disease data were collected during the 5 days before harvest. The condition of leaves near each inoculated fruit was tabulated as healthy, missing or stricken, then the fruit were removed, returned to the laboratory and examined for hull infections and fungal growth.

The influence of hull split size on infection was examined in treatments six, seven and eight. Fruit with small (0.04-0.20 inch), medium (0.20-0.50 inch) and large ( $>0.50$ inch) hull splits, measured at the widest point of separation, were inoculated with $10^{4}$ spores per ml suspension of $R$. stolonifer on the second, third and fourth inoculation dates. The first inoculation date was excluded because there were no fruit with large hull splits. Percent of hull moisture content was measured in 10 fruit from each of the three hull split sizes from each replication on each inoculation date.

Three levels of inoculum concentration were tested in 1991 for their effects on hull infection and leaf death. Fruit that had small- to medium-sized hull splits, in treatments four through eight, were inoculated with $10^{3}, 10^{4}$ or $10^{5}$ spores per $\mathrm{ml}$ suspensions of $R$. stolonifer. One group of 25 fruit with medium split hulls in each replication served as a noninoculated control for this experiment and the hull split size experiment described above.

Weather data were obtained from a CIMIS station located approximately 2.5 miles from the orchard.

\section{Hull rot and irrigation}

Hull rot incidence was affected by irrigation cut-off and hull split size, but not by inoculum concentration.

Natural infection. Hull rot caused by natural infection was reduced in early irrigation cut-off treatments (one through six) and was most severe in later treatments (seven and eight), in which one or two irrigations were made during the last two weeks before harvest (table 1). This distinct delineation between the earliest six and last two treatments was noted in both

\begin{tabular}{|c|c|c|c|c|}
\hline \multirow{2}{*}{$\begin{array}{l}\text { Irrigation cut-off' } \\
\text { treatments }\end{array}$} & \multicolumn{4}{|c|}{ Average predawn leaf water potentialt } \\
\hline & July 26 & August 2 & August 9 & August 16 \\
\hline & & & & \\
\hline 1 & -32 & -33 & -33 & -35 \\
\hline 2 & -30 & -33 & -33 & -32 \\
\hline $\begin{array}{l}3 \\
4\end{array}$ & $\begin{array}{l}-38 \\
-22\end{array}$ & $\begin{array}{r}-33 \\
-38\end{array}$ & -33 & -36 \\
\hline 5 & .22 & $\begin{array}{l}-20 \\
-22\end{array}$ & $\begin{array}{l}-31 \\
-27\end{array}$ & $\begin{array}{l}-31 \\
-30\end{array}$ \\
\hline 6 & -7 & .11 & -22 & .25 \\
\hline 7 & .7 & -12 & -6 & -14 \\
\hline 8 & -8 & -13 & -9 & -7 \\
\hline
\end{tabular}

-Irrigation cut off at weekly intervals beginning 52 days (treatment 1 ) and ending 4 days (treatment 8 ) before harvest.

tReadings are mean values of single leaf measurements on each of four trees per treatment in one replication.

TABLE 3. Effect of hull split size and inoculum concentration on hull rot caused by Rhizopus spp., Kern County, 1991

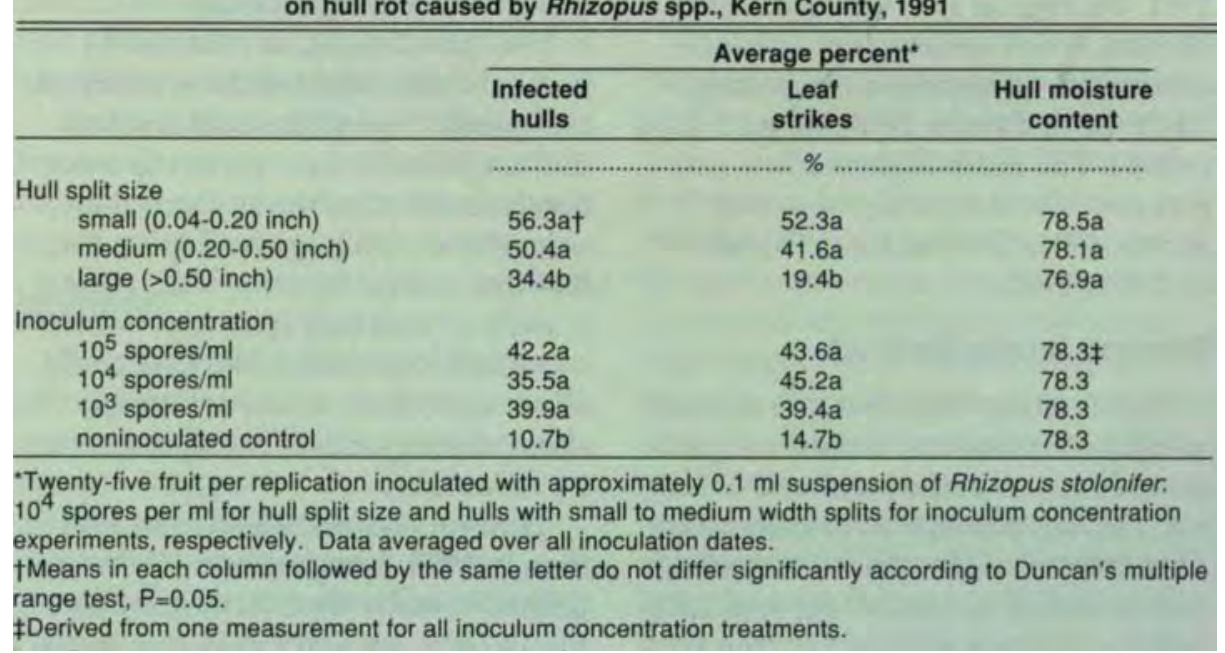


years for percentage hulls infected and number of strikes per tree, and also for estimated inches of dead wood per tree in 1991. However, neither percentage hull moisture content nor percentage split responded consistently.

Predawn leaf water potentials fell below -20 bars within 11 days of water deprivation in early treatments (table 2). A week before harvest, trees in later treatments were less stressed - registering values of -6 and -9 bars - than those measured in earlier treatments. Fully irrigated almond trees have predawn leaf water potentials of -4 to -7 bars. Only trees in the latest irrigation cut-off treatment (treatment eight) maintained low predawn leaf water potentials through harvest.

Hull split size and inoculum concentration. Fruit with large hull splits had fewer infected hulls and strikes, but not lower hull moisture content, than did fruit with small or medium hull splits (table 3 ). Inoculum concentration, excluding the noninoculated control treatment, did not affect the percentage of infected hulls or strikes (table 3).

Average daily maximum and minimum summer temperatures were higher in 1990 than in 1991. Through the course of the irrigation cut-off experiment, mid-June through mid-August, there were 56 and 38 days in 1990 and 1991, respectively, when temperatures rose above $90^{\circ} \mathrm{F}$, and 16 and 6 days, respectively, when they rose above $100^{\circ} \mathrm{F}$. The high and low means for that period were $97.7^{\circ} \mathrm{F}$ and $63.5^{\circ} \mathrm{F}$ in 1990 and $91.4^{\circ} \mathrm{F}$ and $60.4^{\circ} \mathrm{F}$ in 1991. During the five weeks before harvest, when our experiments were conducted, temperatures exceeded $100^{\circ} \mathrm{F}$ on 12 days in 1990 and on 2 days in 1991. The mean high and low temperatures for the period were $98.8^{\circ} \mathrm{F}$ and $66.4^{\circ} \mathrm{F}$ in 1990 and $92.7^{\circ} \mathrm{F}$ and $61.2^{\circ} \mathrm{F}$ in 1991.

\section{Disease levels lowered}

Terminating irrigation two or more weeks before harvest caused a significant reduction in the incidence of hull rot. This suppression of disease might have been related to any of several elements, including percentage hull split and moisture content, inoculum pro- duction and spread, or toxin production and transport. Differences in percentage hull split and hull moisture content did not account for the reduced levels of hull rot. The low percentages of split hulls found in irrigation cut-off treatments one through three may have contributed to the low levels of disease found in those, but in treatments four through eight hull split surpassed $70 \%$ three weeks before harvest. Thus most fruit were susceptible to infection in four, five and six, as well as seven and eight, yet only treatments seven and eight exhibited severe symptoms. Although hull moisture content may prove to influence the growth of $R$. stolonifer or the production of toxin, the small changes encountered among our treatments did not affect hull rot incidence.

The production and dissemination of inoculum might have varied among the treatments and consequently affected disease levels. There were obvious differences in tree condition among the treatments. Early treatment plots were dry, dusty and hot, and the trees showed the usual water stress symptoms consistent with the magnitude of the predawn leaf water potentials. These conditions may have been detrimental to the growth and sporulation of Rhizopus spp. Trees in the latest treatments were most lush, and their dense canopies provided more shade and a cooler environment. In addition, there was more weed growth under these trees. Insects are suspected vectors of the hull rot fungi, and climate might affect their biology and movement to the trees.

Hull attachment, as reflected by hull split size, affected the severity of leaf death. Fruit with small and medium split hulls were generally more firmly attached to the pedicels than were those with large hull splits. Factors that extend the time hulls remain at early or mid hull split, and therefore more fully attached to the tree, could allow more toxin to move into the shoot, thereby exacerbating the severity of hull rot.

Hull rot was more severe in 1991 than in 1990. Symptoms in 1990 were limited to individual strikes, and we found only three strikes in our inocu- lation experiments, all located in treatment eight. Collapsed shoots and small limbs as well as individual strikes were present in 1991. In both years most symptoms developed within the last two weeks before harvest. The high ambient air temperatures in 1990 (56 days with mean temperatures above $90^{\circ} \mathrm{F}$ ) perhaps intensified water stress, and this may have affected hull split, the abscission process, and the transport of toxin. The pathogen, too, may have been limited by the hot weather. The reported maximum growth temperature for $R$. stolonifer is $95^{\circ} \mathrm{F}$.

Manipulation of irrigation could be an important management tool for the control of hull rot. However, our results in Kern County may not apply to other orchard situations. The experimental orchard was planted on shallow soil with a relatively low waterholding capacity so water stress quickly followed water deprivation. Hull rot may not be as easily managed by cessation of irrigation in orchards on deeper soils, because trees will be able to obtain water from lower soil depths in the absence of irrigation. Less stringent deficit irrigation schemes that limit water over the irrigation season rather than summarily cut it off also may not be effective. These situations are the subjects of further investigation.

Beth Teviotdale is Extension Specialist, Plant Pathology, Themis Michailides is Associate Professor, Department of Plant Pathology and David A. Goldhamer is Extension Specialist, Department of Land, Air and Water Resources, UC Davis. All three are based at Kearney Agricultural Center, Parlier. Mario Viveros is Farm Advisor, Kern County. Lisa Schmidt and Valeshia Hines were participants in the UC Davis Summer Undergraduate Affirmative Action Program, Kearney Agricultural Center, Parlier.

The authors wish to thank the Almond Board of California for its generous support of this project, Paramount Farming Company for the use of their orchard, Carol Adams for assistance with statistical analysis, and Dennis Harper, Don Self, Peggy Schrader and Woody Affleck for their technical assistance. 